\title{
Loss of hif- 1 promotes resistance to the exogenous mitochondrial stressor ethidium bromide in Caenorhabditis elegans
}

\author{
Muntasir Kamal ${ }^{1,3}$, Dayana R. D'Amora ${ }^{1}$ and Terrance J. Kubiseski ${ }^{1,2^{*}}$
}

\begin{abstract}
Background: Mitochondrial dysfunction is one of the leading causes of neurological disorders in humans. Mitochondrial perturbations lead to adaptive mechanisms that include HIF-1 stabilization, though the consequences of increased levels of HIF-1 following mitochondrial stress remain poorly understood.

Results: Using Caenorhabditis elegans, we show that a hif-1 loss-of-function mutation confers resistance towards the mitochondrial toxin ethidium bromide (EtBr) and suppresses EtBr-induced production of ROS. In mammals, the

PD-related gene DJ-1 is known to act as a redox sensor to confer protection against antioxidants and mitochondrial inhibitors. A deletion mutant of the C. elegans homolog djr-1.1 also showed increased resistance to EtBr. Furthermore, our data implicates p38 MAP kinase as an indispensable factor for survival against mitochondrial stress in both hif-1 and djr-1.1 mutants.

Conclusions: We propose that EtBr-induced HIF-1 activates pathways that are antagonistic in conferring protection against EtBr toxicity and that blocking HIF-1 activity may promote survival in cells with compromised mitochondrial function.
\end{abstract}

Keywords: C. elegans, DJ-1, Hypoxia inducible factor, Mitochondria, p38 MAPK

\section{Background}

Cells depend on mitochondria to generate the energy required to carry out vital cellular processes. Specific mutations and environmental toxins that target mitochondria are responsible for many degenerative disorders in humans, such as Parkinson's disease (PD), dementia, Alzheimer's disease and mitochondrial myopathy [1]. Mitochondrial dysfunction usually occurs as a result of the organelle's failure to handle stress resulting from normal processes of energy production, due to the loss of one or more mitochondrial protein subunits via deletions or point mutations of mitochondrial DNA (mtDNA), mutations in nuclear DNA that encodes mitochondrial proteins, or the loss of protective mechanisms that ensure maintenance of a

\footnotetext{
* Correspondence: tkubises@yorku.ca

${ }^{1}$ Department of Biology, York University, Toronto, Canada

${ }^{2}$ Department of Neuroscience, York University, Toronto, Canada

Full list of author information is available at the end of the article
}

viable environment to allow these essential processes to occur $[2,3]$.

Loss of mitochondrial electron transport chain (ETC) subunits or mtDNA results in increased ROS production, and this activates antioxidant defense systems. Interestingly, the transcription factor hypoxia inducible factor-1 (HIF-1), which is usually stabilized during hypoxic conditions (conditions of low oxygen concentration) as a protective mechanism, is also stabilized in $C$. elegans with perturbed mitochondria [4]. In mammals, HIF-1 in hypoxic situations plays an important role in hypoxia resistance by continuing to provide the cell with energy from sources that do not require oxygen [5]. Not surprisingly, many tumours exhibit hypoxic environments and thus rely on functional HIF-1 to survive [6]. Importantly, this HIF-1-dependent pathway is favored if mitochondrial function is blocked, since energy production will then depend on anaerobic pathways. A hallmark of many cancer cells is mitochondrial dysfunction accompanied by a hyperactive HIF-1 [7]. Unfortunately, 
the role of HIF-1 in the pathogenesis of mitochondrial diseases is much less understood, partly due to the fact that severe mitochondrial perturbations readily lead to apoptosis in vitro, making long-term studies difficult. Furthermore, most HIF-1 research focuses on mitochondrial perturbation predominantly in the context of tumourigenesis, rather than on mitochondrial diseases themselves.

In this study, we used C. elegans to examine the role of HIF-1 on the protective responses activated when mitochondrial function is impaired and report that in contrast to wild type animals, hif-1 loss-of-function mutants exhibited high resistance to the mitochondrial toxin Ethidium Bromide (EtBr). Since mammalian HIF-1 was shown previously to be downstream of the PD associated gene product DJ-1 [8], we tested a strain containing a deletion of the nematode ortholog djr-1.1 and found that it was also resistant to EtBr. Furthermore, the C. elegans p38/MAPK pathway was strongly induced in $\mathrm{EtBr}$, and was indispensable for growth in both hif-1 and djr-1.1 mutants, indicating that this pathway is involved in protective changes upon mitochondrial perturbations. Taken together, our study shows a role for C. elegans hif-1 in regulating growth and survival during mitochondrial perturbation, and that the C. elegans p38/MAPK cascade is an essential component of a protective response pathway that is activated when mitochondrial function is compromised.

\section{Results}

hif-1 and djr-1.1 mutants are resistant to EtBr exposure compared to wild type worms

The nematode C. elegans undergoes a series of larval developmental stages. The transition from L3 to L4 is associated with a five-fold increase in mtDNA content, and this increases an additional six-fold in transitioning from L4 to adult, as a result of germline expansion [9]. Various studies have shown that one of the effects of mitochondrial perturbation on the health of worms is the reduction of brood size, or fecundity [9-11]. Furthermore, in mammalian cells, it has been shown that $\mathrm{EtBr}$ intercalates between the base pairs of double-stranded DNA and inhibits transcription and replication $[12,13]$. The effect of $\mathrm{EtBr}$ is mostly impairment of mitochondrial DNA and $\mathrm{EtBr}$ is used to rid cells of mitochondrial DNA to create $\rho^{\circ}$ cells [14]. Furthermore, in C. elegans, EtBr has been shown to activate the mtUPR as evidenced by the increase expression of hsp-60, the mitochondrial chaperone that responds to mitochondrial dysfunction upon $\mathrm{EtBr}$ exposure $[15,16]$. Although it is possible that EtBr has some effect on nuclear DNA, it is negligible compared to its effect on the mitochondria [17].
In order to test the roles of hif-1 and djr-1.1 in maintaining the health of worms in which mitochondrial function is impaired by $\mathrm{EtBr}$, we compared the brood sizes of the loss-of-function mutants hif-1 and djr-1.1 to that of the wild type (N2) grown on EtBr plates. We initially determined the brood size of these strains under normal conditions, and found that hif- 1 mutant animals had a lower brood size than N2 (Table 1). In contrast, wild type worms grown on plates containing $25 \mu \mathrm{g} / \mathrm{mL}$ $\mathrm{EtBr}$ had a greatly reduced fecundity over three generations (F1, F2 and F3) compared to both djr-1.1 and hif1mutants. Indeed, hif-1 mutant animals showed to be significantly resilient over three generations as there was no effect on the fecundity of this strain (Fig. 1a). Demands for reproduction are believed to compromise the worm's ability to resist certain stresses (e.g., hypoxia) [4]. This result shows that increased EtBr resistance is not a consequence of reduced brood size.

Since the brood size of djr-1.1 and hif-1 mutant animals were less affected by $\mathrm{EtBr}$, we tested if the growth of these animals was different from that of wild type worms with $\mathrm{EtBr}$ exposure. Previous studies have reported that exposing embryos to EtBr causes L3/L4 larval arrest, indicating that lower mtDNA content cannot support transition to advanced developmental stages that accompany germline proliferation $[9,18,19]$. Here, we found that whereas nearly $50 \%$ of wild type animals showed L3 larval arrest on $50 \mu \mathrm{g} / \mathrm{ml} \mathrm{EtBr}$, only $25 \%$ djr-1.1 mutants arrested at the L3 stage while almost $100 \%$ of hif-1 mutants were able to reach adulthood (Fig. 1b-h). At higher EtBr doses, hif-1 mutants retained the high $\mathrm{EtBr}$ resistance while djr-1.1 mutants exhibited moderate resistance to $\mathrm{EtBr}$ (Fig. 1b-h). These results indicate that the loss of $d j r-1.1$ and particularly hif-1 confers resistance in worms against EtBr-induced mitochondrial perturbation.

To further our understanding for the basis of hif-1 and djr-1.1 mutants ability to survive in the presence of $\mathrm{EtBr}$, we tested a number of other strains for their ability to grow on worm plates containing $50 \mu \mathrm{g} / \mathrm{ml}$ of $\mathrm{EtBr}$ (Fig. 2a). One of the other strains we tested was the hif1(ia7) mutant and found that it displayed resistance to $\mathrm{EtBr}$, indicating that the phenotype demonstrated with the

Table 1 Brood size of N2 (wild type) and mutant animals

\begin{tabular}{ll}
\hline Strain & Brood size (no. of eggs \pm S.D.) \\
\hline N2 & $229.0 \pm 23.8$ \\
hif-1(ia4) & $149.3 \pm 9.8^{*}$ \\
djr-1.1(tm918) & $263.0 \pm 24.1$
\end{tabular}

Single worms were grown in fresh OP50 plates at $22{ }^{\circ} \mathrm{C}$, and the adult worm was transferred to a fresh plate containing OP50 everyday until the worm stopped laying eggs. Figures are mean \pm standard deviation (S.D.). Results are from three independent experiments $(n \geq 40)$. One-way Anova ( ${ }^{*}$ denotes $p<0.05)$ was done to test for statistical significance in brood size between mutant strains and wild-type using GraphPad Prism 5.0 software 


\section{a}
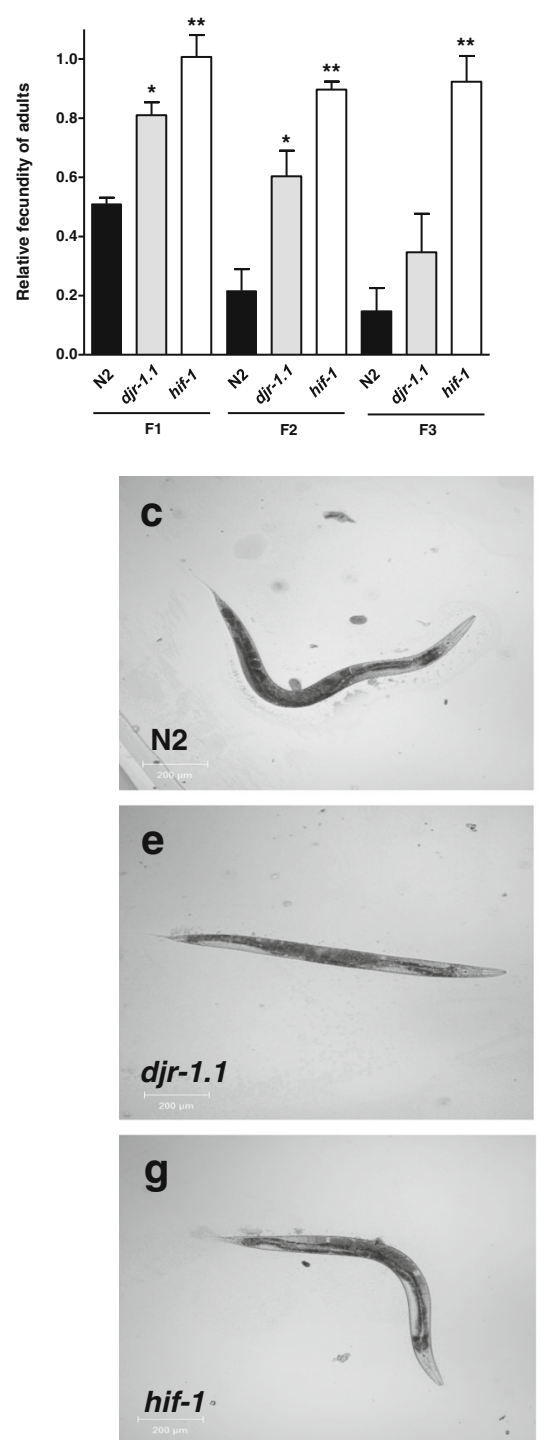

b
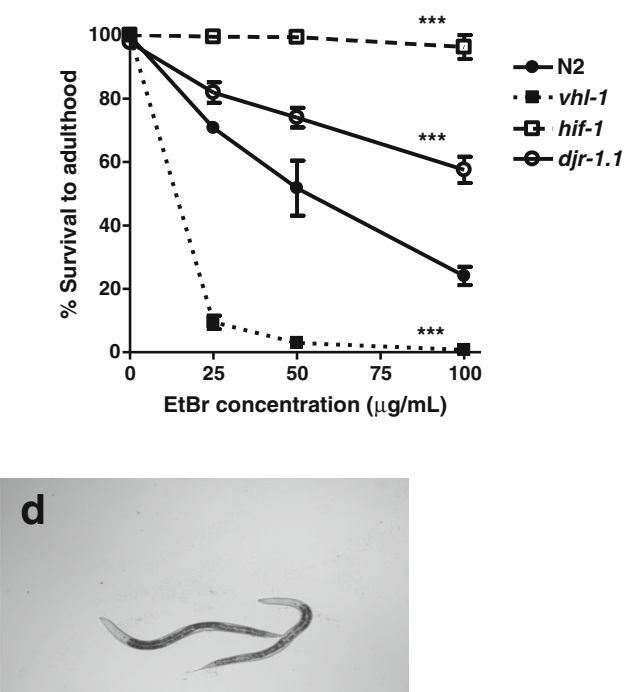

N2 in EtBr
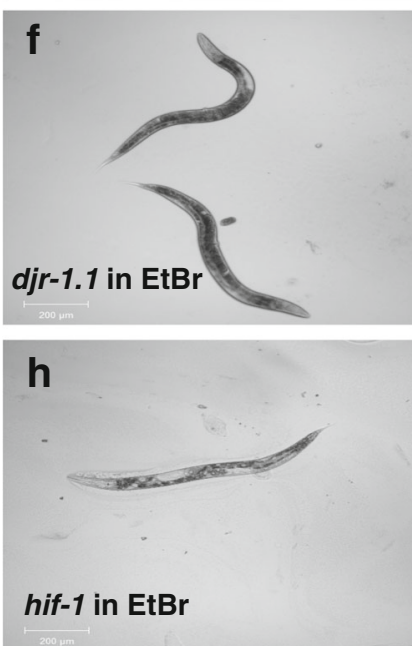

Fig. 1 hif-1 and djr-1.1 mutants are highly resistant to EtBr. a hif-1 animals exposed to EtBr have no effect on fecundity over three generations, while wild type worms showed a significant decrease in brood size. Brood size of N2 (wild type), djr-1.1 and hif-1 animals in $25 \mu \mathrm{g} / \mathrm{mL}$ EtBr were counted for three generations: F1, F2 and F3. Relative fecundity is measured as the number of progeny grown to adulthood on EtBr divided by the number of progeny grown to adulthood in the absence of EtBr, and would include animals that arrest at the L3 stage. Animals with ruptured vulva were not counted. Asterisk marks denote significant difference of fecundity between P0 (normalized to 1.0) and the following generations for each strain (* denotes $p<0.05$ and ** $p<0.01$ ) using one-way Anova. b Concentration dependence of EtBr. N2, hif-1, vhl-1 and djr-1.1 mutant animals (1st generation) were grown on worm plates containing nematode growth media and various concentrations of $\mathrm{EtBr}(0,25,50 \mathrm{and} 100 \mu \mathrm{gg} / \mathrm{mL})$. The number of worms that grew to adulthood was determined and plotted. To determine significant differences between strains, the data were subjected to two-way ANOVA comparing groups (different strains) over different concentrations of EtBr. All strains showed a significant difference compared to N2 (*** $p<0.001)$. c-h Growth of strains on EtBr. N2, djr-1.1 and hif-1 worms (as labeled) four days after egg-laying in plates containing either no EtBr (c, e, g) or $50 \mu \mathrm{g} / \mathrm{mL} \operatorname{EtBr}(\mathbf{d}, \mathbf{f}, \mathbf{h})$

hif-1(ia4) mutant in Fig. 1 was not due to a background mutation. VHL-1 is the worm ortholog of the mammalian Von-Hippel Lindau tumour suppressor [20] and loss-offunction $v h l-1$ stabilizes HIF-1 [21]. We found that the $v h l$ 1 mutant strain demonstrated a very marked sensitivity to $\mathrm{EtBr}$ (Fig. $1 \mathrm{~b}$ and 2a) probably due to the absence of VHL-1 resulting in HIF-1 stabilization [21]. Furthermore, we found that the hif-1;vhl-1 double mutant exhibits a similar level of tolerance to EtBr to that of the hif-1 single mutant (Fig. 2a).

p38 MAPK is required for survival on EtBr-MAP kinases are activated when mitochondrial function is impaired and are believed to play a protective role in 


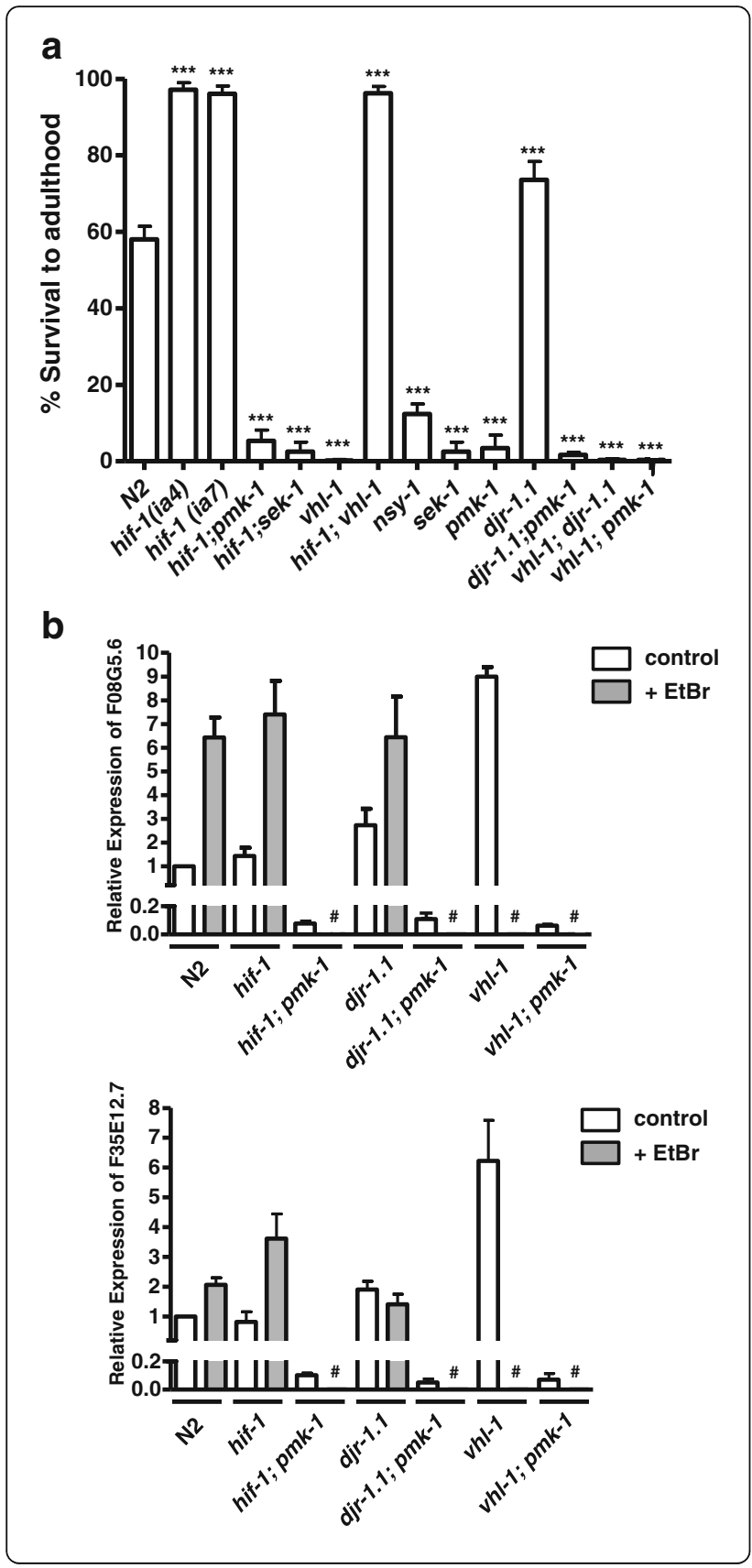

Fig. 2 hif- 1 and djr-1.1 mutants require the nsy-1/sek-1/pmk-1 signalling pathway for EtBr resistance. a pmk-1 is required by hif-1 and djr-1.1 for growth on EtBr containing plates. The first generation of various worm strains containing single or double mutant alleles as listed were grown on worm plates containing nematode growth medium and $50 \mu \mathrm{g} / \mathrm{ml}$ of EtBr, and the percentage of animals that reached the adult stage were determined. Two single mutants of hif-1, hif-1(ia4) and hif-1 (ia7), demonstrated growth in the presence of EtBr. All subsequent experiments were done with the hif-1 (ia4) allele. Furthermore, it was found that the loss of pmk-1 abrogates EtBr resistance of hif-1 and djr-1.1 mutants. Asterisk marks denote significant difference between EtBr-treated N2 and EtBr-treated mutants

(* denotes $p<0.05{ }^{* *} p<0.01,{ }^{* * *} p<0.001$ ). One-way Anova was performed to test for the statistical significant difference in growth between strains. $\mathbf{b}$ pmk-1 is induced in the presence of $\mathrm{EtBr}$ in wild type, hif-1, djr-1.1 and vhl-1 mutants. Transcript levels of two pmk-1 targets (F08G5.6 and F35E12.5) in control $(0 \mu \mathrm{g} / \mathrm{mL}$, "control" open box) and EtBr-treated $(50 \mu \mathrm{g} / \mathrm{mL}$, " $+\mathrm{EtBr}$ "; grey box) samples were measured using qRT-PCR. \# indicates not determined, as vhl-1 mutant and pmk-1 double mutants were unable to survive on worm plates containing EtBr. Results are from three independent experiments

stressed organisms [22-24]. The C. elegans stress-activated kinase PMK-1 is involved in innate immunity and provides protection to bacterial pathogens and oxidative stress [25-27]. Therefore, we asked if the p38/PMK-1 pathway was required for survival on EtBr. Indeed, $n s y-1$, sek-1 and pmk-1 mutants were all extremely sensitive to $\mathrm{EtBr}$, with almost $80 \%$ and close to $100 \%$ of the population undergoing larval arrest for $n s y-1$ and sek1 and $p m k-1$, respectively (Fig. 2a). Importantly, pmk1 was also required for the increased resistance of djr-1.1 and hif-1 animals, with hif-1 also requiring sek-1 for its resistance (the effect of sek-1 mutation on djr-1.1 was not tested): djr-1.1; pmk-1, hif-1;pmk-1 and hif-1;sek-1 double mutants were all highly susceptible to EtBr, similar to pmk-1 and sek-1 single mutants. Although the requirement of $n s y-1$ for djr-1.1 and hif-1 phenotypes was not tested, our data suggest that the nsy-1/sek-1/pmk-1 cascade is required for protection against EtBr toxicity.

Having demonstrated that pmk-1 was required for EtBr resistance, we asked whether pmk-1 was activated upon $\mathrm{EtBr}$ exposure. We tested this by quantifying the expression of two genes, F08G5.6 and F35E12.5, previously shown to be targets of $p m k-1$ but with no clear function $[26,28]$. We reproduced the earlier finding that loss of $d j r-1.1$ results in higher pmk-1 activation (i.e., overexpression of these genes) under normal conditions [26] (Fig. 2b). Notably, pmk-1 was strongly activated in both wild type and djr-1.1 and hif-1 mutant animals in EtBr, except for F35E12.5 in djr-1.1 animals; this suggests that the already high expression of this gene in the djr-1.1 mutant is sufficient to respond to EtBr. In general, the EtBr-induced pmk-1-activation in these mutants was similar in magnitude to that of wild type worms 
(Fig. 2b). As a control, we generated double mutants of pmk-1 with hif-1 and $d j r-1.1$, resulting in a complete inability of the two reporter genes to express in the presence or absence of $\mathrm{EtBr}$, demonstrating that the expression of the reporter genes were dependent on PMK-1 activity (Fig. 2b). Surprisingly, vhl-1 mutant worms also demonstrated higher PMK-1 activity, at least with respect to the genes tested (Fig. 2b). Therefore, although higher PMK-1 activity is required, enhanced PMK-1 activity itself is not sufficient for growth in the presence of EtBr.

ROS levels are lower in hif-1 than in wild type nematodes exposed to EtBr

Previous studies have indicated that mitochondrial perturbation results in elevated ROS levels in the mitochondria, which might further compromise the organelle's ability to handle ROS [29-31]. To measure in vivo ROS levels, we used the compound $\mathrm{H}_{2}$ DCF-DA (2', 7'-dihydrochlorofluorescein diacetate) [29]. We suspended L4/ adult worms treated with either EtBr or water in M9 buffer containing $\mathrm{H}_{2}$ DCF-DA and measured whole organism fluorescence intensity by confocal microscopy. We found that wild type and, to a lesser extent, djr-1.1 worms, had elevated ROS levels, while the EtBr-treated hif-1 animals showed no significant increase (Fig. 3a). This indicates that the presence of a functional hif-1 gene and protein leads to higher endogenous ROS levels upon EtBr treatment.

The gene encoding the mitochondrial superoxide dismutase, sod-3 is overexpressed in EtBr-treated animals indicating elevated ROS levels in these animals [31]. Interestingly, it was reported that DAF-16 translocates to the nucleus when hif- 1 is knocked down by RNAi [32]. Since sod-3 is a well-known target for DAF-16 [33], we tested if sod-3 transcript levels differed in the mutant worms from that of wild type following EtBr exposure. Under normal conditions, sod-3 transcript levels were slightly higher in hif-1 animals, while sod-3 levels in djr1.1 and wild type were similar (Fig. 3b). EtBr exposure resulted in sod-3 overexpression in wild type and $d j r-1.1$ worms, but we found no significant change in expression in EtBr-treated hif-1 samples. This was confirmed by increased sod-3::gfp expression in EtBr-treated wild type animals but no increase in sod-3::gfp expression in hif-1 animals (Fig. 3c-f). Taken together, our data suggest that ROS levels and hence, oxidative stress, is lower in $\mathrm{EtBr}$ treated hif-1 animals.

\section{The hif-1-dependent gene tyrosinase tyr-2/TRP2 is overexpressed in $\mathrm{EtBr}$}

Previously, it was shown that EtBr activates the expression of well-established hif-1 dependent genes such as $n h r-57$ and cysl-2 [11]. To confirm that in our system we were seeing an increase in the expression of hif-1 dependent genes upon exposure to $\mathrm{EtBr}$, we looked at the expression of tyr-2. The C. elegans tyr-2 gene was first reported to be overexpressed with hypoxia treatment ( $0.5 \%$ oxygen) in a hif-1-dependent manner [34], and was later shown to be responsible for the antiapoptotic property of HIF-1 by suppressing cep-1/p53dependent germline apoptosis following ionizing radiation [21]. hif-1 mutants exhibit very low tyr-2 mRNA transcript levels, while vhl-1 mutants show higher tyr-2 transcript levels, which is consistent with the idea that tyr-2 regulation is hif-1 dependent [21]. Here, we found that tyr-2 was overexpressed in EtBr-treated samples, showing an almost two-fold increase in EtBr treated wild type and djr-1.1 samples that did not differ between the two strains (Fig. 4). As expected, tyr-2 expression did not increase in EtBr-treated hif-1 mutant samples (Fig. 4). These results confirm the previous report [11] that HIF1 gene expression activity occurs in the presence of $\mathrm{EtBr}$ in wild type worms.

\section{Discussion}

This study was carried out to investigate the role of HIF-1 in survival of worms subjected to mitochondrial stress. Previous results have indicated that overexpression of HIF-1 in C. elegans promotes longevity in a manner parallel to SKN-1/NRF and DAF-16/FOXO transcription factors [35]. Here, we found that hif-1 mutants grew significantly better on $\mathrm{EtBr}$ compared to wild type worms, indicating that the hif-1-dependent response activated in $\mathrm{EtBr}$ antagonizes any adaptive mechanism required for $\mathrm{EtBr}$ tolerance. Furthermore, our results show for the first time to our knowledge that the p38 MAPK pathway is strongly induced in worms with compromised mitochondrial function, and that pmk-1 is indispensable for $\mathrm{EtBr}$ survival. Finally, we also show that ROS levels are lower in hif- 1 worms, indicating that p38/PMK-1 activity and lower oxidative stress in hif- 1 mutant animals probably both contribute to promote survival in the presence of EtBr.

In C. elegans, inactivation of genes encoding subunits of the mitochondrial respiratory chain can stabilize HIF1 , and a hif-1-dependent hypoxia response is induced in animals with compromised mitochondrial function [36]. Because mitochondrial ROS generation is increased in these animals and because mitochondrial ROS stabilizes HIF-1 during hypoxia, a hypoxia response may be initiated during mitochondrial stress that may not necessarily be specific to hypoxia but modified to act as a survival pathway specific to mitochondrial stress. Alternatively, it is possible that hif-1-induced pathways may have pleiotropic effects on survival, as a consequence of hypoxia-specific pathways being altered during mitochondrial stress when not accompanied by true hypoxic condition. We did not test if a full hif-1 dependent hypoxia response or at least specific genes in the hypoxia pathway which 

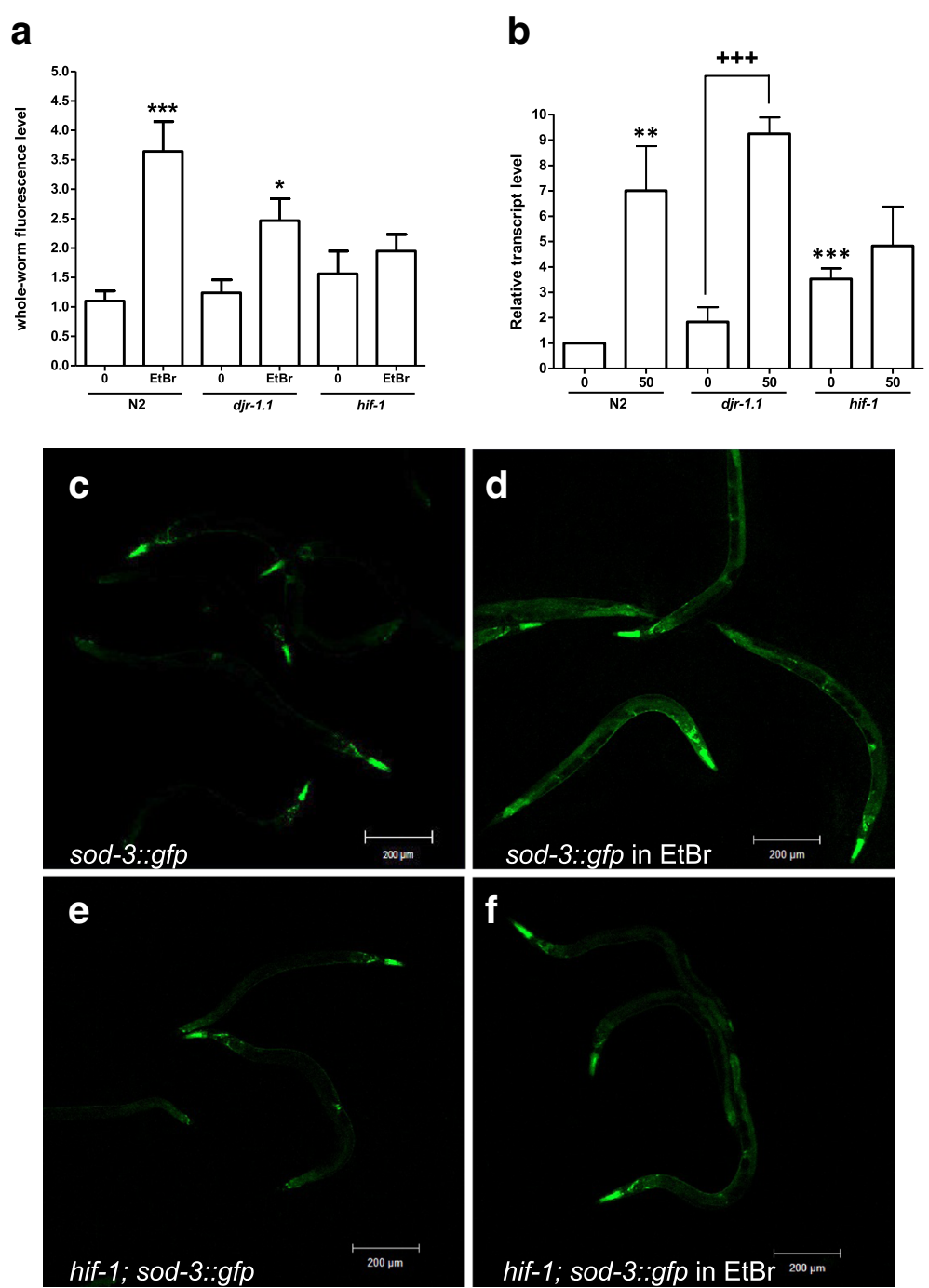

Fig. 3 ROS production is enhanced in EtBr-treated wild type and djr-1.1 worms, but not hif-1 mutant worms. a In vivo ROS levels were determined in L4/adult worms grown on worm plates containing either $\mathrm{EtBr}(50 \mu \mathrm{g} / \mathrm{mL})$ or water. Worms were subsequently incubated in buffer containing $\mathrm{H}_{2}$ DCF-DA and whole organism fluorescence intensity measured using a Zeiss LSM 700 confocal microscope. Results were summarized from at least 4 independent experiments. Fluorescence intensity was quantified using ImageJ software. The y-axis depicts absolute values of mean intensity per worm. Statistical analysis was performed in GraphPad Prism 5.0. Asterisk marks denote significant difference between control and drug-treated worm samples for each strain $\left({ }^{*} p<0.05,{ }^{* *} p<0.01,{ }^{* * *} p<0.001\right)$. b sod-3 is overexpressed in EtBr-treated wild type and djr-1.1 animals but not EtBr-treated hif-1 animals. mRNA levels of sod-3 was determined by qRT-PCR in wild type, hif-1 and djr-1.1 mutants grown on worm plates containing nematode growth media in either the absence (labeled 0) or presence (labeled 50) of $50 \mu \mathrm{g} / \mathrm{ml}$ of EtBr, using act-1 as a control. Asterisk/plus signs indicate statistical significant difference in expression between control N2 sample and other strains and that between untreated and EtBr-treated sample for each strain $(* /+$ denotes $p<0.05, * * /++p<0.01, * * *+++p<0.001)$. sod-3 transcript levels were significantly higher in wild type and djr-1.1 animals, but not hif-1 mutants. c Wild type sod-3::gfp and hif-1;sod-3::gfp strains adults were placed on plates containing EtBr and images were taken of five to 10 worms three and four days post egg-laying for hif-1;sod-3::gfp and wild type animals, respectively

might determine the EtBr phenotype were induced; thus, it will be interesting to test if mutations in hif-1-dependent hypoxia signalling pathways confer worms with a similar resistance towards EtBr.

Our findings add to the growing body of evidence that suggests the consequence of HIF-1 stabilization will depend on the type and/or severity of perturbation: for example, with mitochondrial stress produced in ETC. point mutants [36] or diluted ETC (by RNAi knockdown) animals [37], HIF-1 may have a chiefly beneficial or a protective function and is required for lifespan extension. But in other types of stress, such as from an exogenous stressor like EtBr, the same or additional HIF-1-independent pathways are activated that reduce mitochondrial stress. In this case, HIF-1 activation is likely to be "reactive" in nature, and may activate pathways that are superfluous, or even 


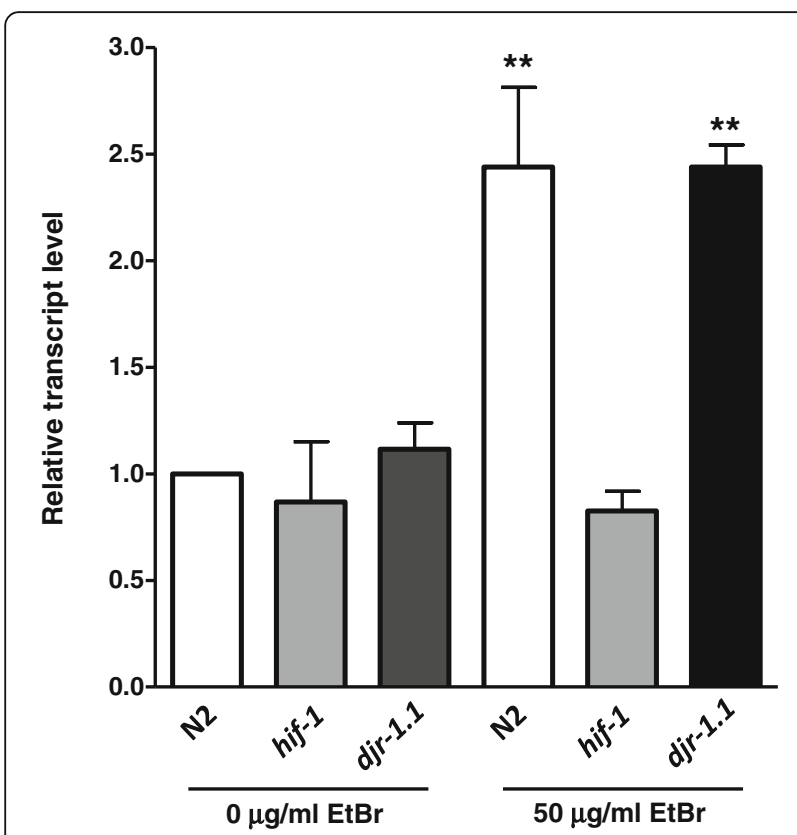

Fig. 4 The hif-1-dependent gene tyr-2 is overexpressed in wild type and djr-1.1 mutants but not in hif-1 animals treated with EtBr. The transcript level of tyr-2 was determined by qRT-PCR in wild type, hif-1 and djr-1.1 mutant worms grown on worm plates in the absence and presence of $50 \mu \mathrm{g} / \mathrm{ml}$ of EtBr. The experiment was performed independently at least three times. GraphPad Prism 5.0 was used to find statistical significance. Asterisk marks denote statistical significant differences ( ${ }^{*}$ denotes $p<0.05,{ }^{* *} p<0.01,{ }^{* * *} p<0.001$ ) in tyr-2 expression in wild type and djr-1.1 between untreated and EtBr treated samples but not in hif-1 animals

antagonistic to mitochondrial-stress specific pathways. We propose that such inappropriate effects of HIF-1 activation are a direct consequence of its complex, pleiotropic role on cellular stress response in general.

In mammals, the gene $D J-1$ encodes a protein that can act as a redox-sensor, and can act as an antioxidant during oxidative stress and is upstream of HIF-1 [8]. C. elegans has two homologues, djr-1.1 and djr-1.2. Surprisingly, we found that $d j r-1.1$ mutants were significantly more resistant to EtBr than wild type worms, a phenotype it shares with hif-1, indicating that these two genes probably share overlapping functions. The NSY-1/ SEK-1/PMK-1 pathway confers innate immunity to worms against bacterial pathogens, and djr-1.1 mutation potentiates this signalling [26]. Therefore, activation of immune signalling via NSY-1/SEK-1/PMK-1 may increase the survival of worms against various stresses including perturbation of mitochondrial function and djr-1.1 worms probably had greater survival in EtBr because of higher $p m k-1$ activation under normal conditions. However, since djr-1.1 mutants were susceptible to a higher $\mathrm{EtBr}$ dose, it is conceivable that the limit of pmk-1 activation in these animals restricts their ability to resist EtBr toxicity.
Our results have implicated the p38 MAPK pathway as an important factor in regulating survival against mitochondrial stress. In agreement with our finding, Liu et al. [38] reported worms interpret mitochondrial perturbation as a xenobiotic/pathogen infection, eliciting a xenobiotic and pathogenic response. Since the ASK-1/ MKK3/6/p38 MAPK pathway is specifically responsible for triggering apoptotic cell death and neuronal loss in PD, it will be important to precisely identify downstream targets of p38 MAPK that are activated upon EtBr exposure that are essential for survival in $\mathrm{EtBr}$, so that pharmacological intervention to enhance the activity of those genes can slow down or improve pathogenesis of PD and other mitochondrial diseases.

\section{Conclusions}

We propose that HIF-1 activates pathways upon exposure to the mitochondrial disrupter $\mathrm{EtBr}$ that hinders survival of cells experiencing this type of mitochondrial stress. Many tumour tissues exhibit pathological hypoxic conditions and have a hyperactive HIF-1 [39]. Tumour cells also tend to have impaired mitochondrial function, such that HIF-1 makes the appropriate metabolic shift to anaerobic respiration that ultimately contributes to the cancer phenotype [40]. Consequently, current cancer treatments target HIF-1 in order to force cancer cells to depend on the dysfunctional mitochondria, in the hope that cancer cells would then fail to grow and ultimately be out-competed by normal cells. We propose the possibility that HIF-1-dependent gene expression might hinder survival in cells experiencing mitochondrial stress so that loss of HIF-1, especially in normoxic cancer cells, might actually be a benefit to cells. However, since worms do not have a HIF-2 homologue, the role of HIF-1 in worms may differ in significant ways from that in more complex organisms. Therefore, it will be important to validate the findings of this study in other model organisms.

\section{Methods \\ C. elegans strains}

Worm strains were provided by either the Caenorhabditis Genetics Center (CGC, University of Minnesota, Twin Cities, USA) or by the Mitani Lab through the National Bio-Resource Project of the MEXT, Japan. All the worms were outcrossed a sufficient number of times prior to order to ensure a uniform genetic background. All double mutants were created using standard $C$. elegans techniques and confirmed by single worm PCR to identify deletions when possible. Primers used for genotyping and qRT-PCR are available upon request. The N2 (Bristol) is the wild type worm strain, and other strains used were: AU3 [nsy-1(ag3)], CF1553 [muIs84(sod-3::gfp)], KU4 [sek-1(km4)], KU25 [pmk-1(km25)], YF134 [hif-1(ia4); sek-1(km4)], YF136 [djr-1.1(tm918)], YF137 [hif-1(ia4); 
vhl-1(ok161)], YF138 [djr-1.1(tm918); pmk-1(km25)], YF143 [hif-1(ia4); muIs84(sod-3::gfp)], YF149 [hif-1(ia4); pmk1(km25)], YF154 [vhl-1(ok161)], YF179 [djr-1.1(tm918); vhl-1(ok161)], YF180 [djr-1.1(tm918); pmk-1(km25)], YF 190 [vhl-1(ok161); pmk-1(km25)], ZG31 [hif-1(ia4)] and ZG596[hif-1(ia7)].

\section{Single-worm PCR (SW-PCR)}

Individual worms were placed in PCR tubes containing $4 \mu \mathrm{L} 1 \times$ Thermopol $^{\circ}$ reaction buffer (NEB) and $0.5 \mathrm{mg} / \mathrm{mL}$ proteinase $\mathrm{K}$ buffer (NEB) and incubated at $-80{ }^{\circ} \mathrm{C}$ for $30 \mathrm{~min}$, then incubated at $65{ }^{\circ} \mathrm{C}$ for $1 \mathrm{~h}$ and $95{ }^{\circ} \mathrm{C}$ for 15 min to inactivate proteinase $\mathrm{K}$. SW-PCR was initiated by adding $20 \mu \mathrm{L}$ of PCR mix that contained primer pairs, dNTPs, and $1 \times$ Thermopol $^{\circ}$ reaction buffer for a reaction volume of $24 \mu \mathrm{L}$. The PCR cycle consisted of the following steps: $55{ }^{\circ} \mathrm{C}$ for $60 \mathrm{~s}, 94{ }^{\circ} \mathrm{C}$ for $5 \min 15 \mathrm{~s}, 55^{\circ} \mathrm{C}$ for $15 \mathrm{~s}$, $72{ }^{\circ} \mathrm{C}$ for $1 \mathrm{~min}$ ( 35 cycles), and $72{ }^{\circ} \mathrm{C}$ for $5 \mathrm{~min}$ (total reaction time $1 \mathrm{~h}$ and $30 \mathrm{~min}$ ).

\section{Brood size assay in control and EtBr plates at $22{ }^{\circ} \mathrm{C}$}

Single worms were grown in fresh OP50 plates, and the adult worm was transferred to a fresh plate containing OP50 every day until the worm stopped laying eggs. Total number of eggs laid was recorded by counting number of L1 larvae and unhatched eggs. Brood size of $\mathrm{N} 2$ (wild type), $\operatorname{djr}-1.1$ and hif- 1 animals at $22{ }^{\circ} \mathrm{C}$ in $25 \mu \mathrm{g} / \mathrm{mL}$ EtBr for three generations F1, F2 and F3 were recorded. For each generation, single worms were grown in fresh OP50 plates containing $25 \mu \mathrm{g} / \mathrm{mL} \mathrm{EtBr}$ or double-distilled water (control), and the adult worm was transferred to a fresh plate containing $25 \mu \mathrm{g} / \mathrm{mL}$ EtBr or distilled water seeded with $50 \mu \mathrm{L}$ OP50 every day until the worm stopped laying eggs. For each strain, relative fecundity is defined as the ratio of total number of eggs laid in $\mathrm{EtBr}$ plates to total number of eggs laid in control (no EtBr) plates.

\section{EtBr treatment and survival assay}

To make 25, 50 and $100 \mu \mathrm{g} / \mathrm{mL}$ EtBr plates, $10 \mathrm{mg} / \mathrm{mL}$ original stock $\mathrm{EtBr}$ was diluted with double-distilled water to $0.5,1.0$ and $2.0 \mathrm{mg} / \mathrm{mL}$, respectively. $250 \mu \mathrm{L}$ of diluted $\mathrm{EtBr}$ was added to worm plates for desired $\mathrm{EtBr}$ concentration. The plates were left to dry for two days, after which they were seeded with $50 \mu \mathrm{L}$ OP50. For survival assays, only 50 and $100 \mu \mathrm{g} / \mathrm{mL}$ EtBr were used. Six gravid adults were placed on each of two plates (i.e., duplicates) containing 50 or $100 \mu \mathrm{g} / \mathrm{mL}$ ethidium bromide (EtBr) seeded with $50 \mu \mathrm{L}$ OP50 bacteria and allowed to lay eggs for three hours (Day 1). On Day 2, total number of L1 animals in the two plates after egg-hatching were counted. Between Days 4-6, total number of adults in the two plates were counted and recorded as a percentage of total number of L1's observed on Day 2. The experiment was done three to seven times, with the number of L1's per plate greater than 40 . For each strain, experiments were carried out to record proportion of L1's reaching adulthood in control plates; it was observed that $>99 \%$ of population grew to adulthood in control plates for all strains studied, and thus control plates were not included in the EtBr survival experiments.

\section{Quantitative reverse-transcriptase PCR (qRT-PCR)}

Total RNA was isolated using the standard Trizol method, using manufacturer's instructions. For the PCR step, master mix was prepared with forward and reverse primers $(0.5 \mu \mathrm{g} / \mu \mathrm{L})$ diluted $1 / 10$ in a final volume $40 \mu \mathrm{L}$. They were further diluted by adding $3.7 \mu \mathrm{L}$ of the diluted primers to $25.3 \mu \mathrm{L}$ of RT-PCR grade water for each strain (total volume $29 \mu \mathrm{L}$ ). To this was added $4 \mu \mathrm{L}$ of cDNA, followed by addition of $33 \mu \mathrm{L} \mathrm{SYBR}$ Advantage $^{\circ}$ qPCR Premix (Clontech). act-1 was used as an internal control; $20 \mu \mathrm{L}$ of samples were loaded in 96-well plates in triplicates, and performed with comparative $C_{T}\left(\Delta \Delta C_{T}\right)$ as the quantification method with the following steps: $50{ }^{\circ} \mathrm{C}$ for $20 \mathrm{~s}$ and $95{ }^{\circ} \mathrm{C}$ for $10 \mathrm{~s}$ (holding stage), $95^{\circ} \mathrm{C}$ for $15 \mathrm{~s}$ and $60^{\circ} \mathrm{C}$ for $1 \mathrm{~min}$ (cycling stage, 40 cycles), $95{ }^{\circ} \mathrm{C}$ for $15 \mathrm{~s}, 60{ }^{\circ} \mathrm{C}$ for $1 \mathrm{~min}, 95^{\circ} \mathrm{C}$ for $30 \mathrm{~s}$ and $60^{\circ} \mathrm{C}$ for $15 \mathrm{~s}$ (melt curve stage).

\section{ROS quantification}

For each strain, four gravid adults were allowed to lay eggs for $6 \mathrm{~h}$ in plates containing $50 \mu \mathrm{g} / \mathrm{mL} \mathrm{EtBr}$ and seeded with $50 \mu \mathrm{L}$ OP50 bacteria (Day 1). On Day 4, L4 or adult worms were washed with M9 buffer and incubated in $1 \mathrm{~mL}$ M9 containing $10 \mu \mathrm{M} \mathrm{H} \mathrm{H}_{2}$ DCF-DA (dissolved in DMSO) for $45 \mathrm{~min}$ in the dark. After incubation, the tubes were spun at a low speed (2,000 rpm) and worms were placed on unseeded agar plates. Live worms were then placed on slides with an agarose pad, covered with a cover-slip, and, using confocal microscopy, z-stack analysis was performed with the same exposure, magnification $(20 \times)$ and number of slices (ten) for all individual worms and strains. Alexa Fluor 488 was used as the emission spectrum. Five to ten worms were analyzed for each strain per experiment. Fluorescence intensity was quantified using ImageJ software.

\section{DIC microscopy}

Live worms were placed in a drop of $2 \mathrm{mM}$ levamisole on a slide with an agarose pad and closed with a coverslip. All images were taken using a confocal microscope at $20 \times$ magnification, with default DIC settings.

\section{Abbreviations}

DIC: Differential interference contrast; EtBr: Ethidium bromide; ETC: Electron transport chain; $\mathrm{H}_{2}$ DCF-DA: 2',7'-dihydrochlorofluorescein diacetate; HIF-1: Hypoxia inducible factor 1; mtDNA: Mitochondrial DNA; mtUPR: Mitochondrial unfolded protein response; PCR: Polymerase chain reaction; PD: Parkinson's disease; qRT-PCR: Quantitative reverse transcriptase polymerase chain reaction; ROS: Reactive oxygen species 


\section{Acknowledgements}

Worm strains were provided by the National Bioresource Project, and the CGC, which is funded by NIH Office of Research Infrastructure Programs (P40 OD010440).

\section{Funding}

This work was supported by a Discovery Grant from the Natural Sciences and Engineering Research Council of Canada (NSERC).

\section{Availability of data and materials}

Data available from corresponding author upon reasonable request.

\section{Author contributions}

Conceived and designed the experiments: MK and TJK. Performed the experiments: MK and DRD. Analyzed the data: MK and TJK. Wrote the paper: MK and TJK. All authors read and approved the final manuscript.

\section{Competing interests}

The authors declare that they have no competing interests.

\section{Consent for publication}

Not applicable.

\section{Ethics approval and consent to participate}

Not applicable.

\author{
Author details \\ ${ }^{1}$ Department of Biology, York University, Toronto, Canada. ${ }^{2}$ Department of \\ Neuroscience, York University, Toronto, Canada. ${ }^{3}$ Present address: \\ Department of Molecular Genetics, University of Toronto, Toronto, Canada.
}

Received: 16 December 2015 Accepted: 6 September 2016 Published online: 13 September 2016

\section{References}

1. Hameed S, Hsiung HS. The role of mitochondria in aging, neurodegenerative disease, and future therapeutic options. BC Med J. 2011;53:188-92.

2. Aguilaniu H, Durieux J, Dillin A. Metabolism, ubiquinone synthesis, and longevity. Genes Dev. 2005;19:2399-406.

3. Kaletsky R, Murphy CT. The role of insulin/IGF-like signaling in C. elegans longevity and aging. Dis Model Mech. 2010;3:415-9.

4. Powell-Coffman JA. Hypoxia signaling and resistance in C. elegans. Trends Endocrinol Metab. 2010;21:435-40.

5. Chandel NS. Mitochondrial regulation of oxygen sensing. Adv Exp Med Biol. 2010;661:339-54.

6. Rankin EB, Giaccia AJ. The role of hypoxia-inducible factors in tumorigenesis. Cell Death Differ. 2008;15:678-85.

7. Boland ML, Chourasia AH, Macleod KF. Mitochondrial dysfunction in cancer. Front Oncol. 2013;3:292.

8. Vasseur S, Afzal S, Tardivel-Lacombe J, Park DS, lovanna JL, Mak TW. DJ-1/ PARK7 is an important mediator of hypoxia-induced cellular responses. Proc Natl Acad Sci U S A. 2009;106:1111-6.

9. Tsang WY, Lemire BD. Mitochondrial genome content is regulated during nematode development. Biochem Biophys Res Commun. 2002;291:8-16.

10. Yoneda T, Benedetti C, Urano F, Clark SG, Harding HP, Ron D. Compartment-specific perturbation of protein handling activates genes encoding mitochondrial chaperones. J Cell Sci. 2004;117:4055-66.

11. Sugimoto T, Mori C, Takanami T, Sasagawa Y, Saito R, Ichiishi E, et al. Caenorhabditis elegans par2.1/mtssb-1 is essential for mitochondrial DNA replication and its defect causes comprehensive transcriptional alterations including a hypoxia response. Exp. Cell Res. 2008:314:103-14.

12. Marusich MF, Robinson BH, Taanman JW, Kim SJ, Schillace R, Smith JL, et al. Expression of mtDNA and nDNA encoded respiratory chain proteins in chemically and genetically-derived Rho0 human fibroblasts: a comparison of subunit proteins in normal fibroblasts treated with ethidium bromide and fibroblasts from a patient with mtDNA depletion syndrome. Biochim Biophys Acta. 1997;1362:145-59.

13. Leibowitz RD. The effect of ethidium bromide on mitochondrial DNA synthesis and mitochondrial DNA structure in HeLa cells. J Cell Biol. 1971;51:116-22.

14. Hashiguchi K, Zhang-Akiyama Q-M. Establishment of human cell lines lacking mitochondrial DNA. Methods Mol Biol Clifton NJ. 2009;554:383-91.
15. Haynes CM, Ron D. The mitochondrial UPR - protecting organelle protein homeostasis. J Cell Sci. 2010;123:3849-55.

16. Nargund AM, Pellegrino MW, Fiorese CJ, Baker BM, Haynes CM. Mitochondrial import efficiency of ATFS-1 regulates mitochondrial UPR activation. Science. 2012;337:587-90.

17. Horwitz HB, Holt CE. Specific inhibition by ethidium bromide of mitochondrial DNA synthesis in physarum polycephalum. J Cell Biol. 1971;49:546-53.

18. Ventura N, Rea SL, Schiavi A, Torgovnick A, Testi R, Johnson TE. p53/CEP-1 increases or decreases lifespan, depending on level of mitochondrial bioenergetic stress. Aging Cell. 2009;8:380-93.

19. Addo MG, Cossard R, Pichard D, Obiri-Danso K, Rötig A, Delahodde A. Caenorhabditis elegans, a pluricellular model organism to screen new genes involved in mitochondrial genome maintenance. Biochim Biophys Acta. 1802;2010:765-73.

20. Bishop T, Lau KW, Epstein ACR, Kim SK, Jiang M, O'Rourke D, et al. Genetic analysis of pathways regulated by the von Hippel-Lindau tumor suppressor in Caenorhabditis elegans. PLoS Biol. 2004;2:e289.

21. Sendoel A, Kohler I, Fellmann C, Lowe SW, Hengartner MO. HIF-1 antagonizes p53-mediated apoptosis through a secreted neuronal tyrosinase. Nature. 2010;465:577-83.

22. Emerling BM, Platanias LC, Black E, Nebreda AR, Davis RJ, Chandel NS. Mitochondrial reactive oxygen species activation of p38 mitogenactivated protein kinase is required for hypoxia signaling. Mol Cell Biol. 2005:25:4853-62.

23. Lee J, Song J, Kwon K, Jang S, Kim C, Baek K, et al. Human DJ-1 and its homologs are novel glyoxalases. Hum Mol Genet. 2012;21:3215-25.

24. Butow RA, Avadhani NG. Mitochondrial signaling: the retrograde response. Mol Cell. 2004;14:1-15.

25. Hayakawa T, Kato K, Hayakawa R, Hisamoto N, Matsumoto K, Takeda K, et al. Regulation of anoxic death in Caenorhabditis elegans by mammalian apoptosis signal-regulating kinase (ASK) family proteins. Genetics. 2011;187:785-92.

26. Cornejo Castro EM, Waak J, Weber SS, Fiesel FC, Oberhettinger P, Schütz M, et al. Parkinson's disease-associated DJ-1 modulates innate immunity signaling in Caenorhabditis elegans. J Neural Transm. 2010;117:599-604. Vienna Austria 1996

27. Jones SJ, Riddle DL, Pouzyrev AT, Velculescu VE, Hillier L, Eddy SR, et al. Changes in gene expression associated with developmental arrest and longevity in Caenorhabditis elegans. Genome Res. 2001;11:1346-52.

28. Troemel ER, Chu SW, Reinke V, Lee SS, Ausubel FM, Kim DH. p38 MAPK regulates expression of immune response genes and contributes to longevity in C. elegans. PLoS Genet. 2006;2:e183.

29. Lee H-C, Wei Y-H. Mitochondrial biogenesis and mitochondrial DNA maintenance of mammalian cells under oxidative stress. Int J Biochem Cell Biol. 2005:37:822-34.

30. Lee SS, Lee RYN, Fraser AG, Kamath RS, Ahringer J, Ruvkun G. A systematic RNAi screen identifies a critical role for mitochondria in C. elegans longevity. Nat Genet. 2003;33:40-8.

31. Yang W, Hekimi S. A mitochondrial superoxide signal triggers increased longevity in Caenorhabditis elegans. PLoS Biol. 2010;8:e1000556.

32. Leiser SF, Begun A, Kaeberlein M. HIF-1 modulates longevity and healthspan in a temperature-dependent manner. Aging Cell. 2011;10:318-26.

33. Honda Y, Honda S. The daf-2 gene network for longevity regulates oxidative stress resistance and $\mathrm{Mn}$-superoxide dismutase gene expression in Caenorhabditis elegans. FASEB J. 1999;13:1385-93.

34. Shen C, Nettleton D, Jiang M, Kim SK, Powell-Coffman JA. Roles of the HIF-1 hypoxia-inducible factor during hypoxia response in Caenorhabditis elegans. J Biol Chem. 2005;280:20580-8.

35. Zhang Y, Shao Z, Zhai Z, Shen C, Powell-Coffman JA. The HIF-1 hypoxiainducible factor modulates lifespan in C. elegans. PLoS One. 2009;4:e6348.

36. Lee S-J, Hwang AB, Kenyon C. Inhibition of respiration extends C. elegans life span via reactive oxygen species that increase HIF-1 activity. Curr Biol. 2010;20:2131-6.

37. Rea SL, Ventura N, Johnson TE. Relationship Between Mitochondrial Electron Transport Chain Dysfunction, Development, and Life Extension in Caenorhabditis elegans. PLoS Biol. 2007;5:e259.

38. Liu Y, Samuel BS, Breen PC, Ruvkun G. Caenorhabditis elegans pathways that surveil and defend mitochondria. Nature. 2014;508:406-10.

39. Zhang H, Bosch-Marce M, Shimoda LA, Tan YS, Baek JH, Wesley JB, et al. Mitochondrial autophagy is an HIF-1-dependent adaptive metabolic response to hypoxia. J Biol Chem. 2008;283:10892-903.

40. Hsu PP, Sabatini DM. Cancer cell metabolism: Warburg and beyond. Cell. 2008;134:703-7. 